\title{
Molecular characterization of Klebsiella pneumoniae isolates from stool specimens of outpatients in sentinel hospitals Beijing, China, 2010-2015
}

Bing Lu', Haijian Zhou ${ }^{2,3}$, Xin Zhang ${ }^{1}$, Mei Qu' ${ }^{1}$, Ying Huang ${ }^{1}$ and Quanyi Wang ${ }^{\text {** }}$

\begin{abstract}
Background: Klebsiella pneumoniae (K. pneumoniae) is an opportunistic pathogen associated with communityacquired infections and nosocomial infections. From 2010 to 2015, K. pneumoniae testing was included into the exiting diarrhea-syndrome surveillance with objective to estimate the prevalence of $K$. pneumoniae in diarrhea-syndrome patients, test antibiotics susceptibility and investigate molecular characteristics.

Methods: Stool specimens from diarrhea-syndrome outpatients were cultured and identified the pathogens by the Vitek2 Compact instrument. The isolated K. pneumoniae strains were tested for antibiotics susceptibility by minimal inhibitory concentration (MIC) method, and subtyped by pulsed field gel electrophoresis (PFGE) and multilocus sequence typing (MLST).

Results: 22 K. pneumoniae strains were identified from 4340 stool specimens of outpatients who visited sentinel hospitals in Beijing during 2010-2015. All strains were sensitive to gentamicin, nalidixic acid, ciprofloxacin, ceftriaxone, cefotaxime, cefepime, imipenem. The highest resistance rate of $K$. pneumoniae strains was $100 \%$ to amoxicillin-clavulanate, followed by $72.7 \%$ to ampicillin. These $22 \mathrm{~K}$. pneumoniae strains were characterized into 21 different PFGE types and 20 MLST types with less similarity.

Conclusions: The detection rate of $K$. pneumoniae in stool specimens from outpatients with diarrhea syndromes was about $0.5 \%$ in Beijing. Less similarity of the isolated strains indicated the unlikely long-term circulating of $K$. pneumoniae in the community. ST23 was the most common genotype. Drug resistance of the community-acquired K. pneumoniae was not a serious problem in comparing with hospital-acquired infections. High vigilance in the communityacquired $K$. pneumoniae strains and investigation of pathogens' microbiological characteristics are valuable for signals detection for drug resistance in population and strains variation.
\end{abstract}

Keywords: Klebsiella pneumoniae, Drug resistance, Multilocus sequence typing

\section{Background}

Klebsiella spp. is ubiquitous in nature. Klebsiella pneumoniae (K. pneumoniae) is an opportunistic pathogen associated with both community-acquired and nosocomial

\footnotetext{
*Correspondence: bjcdcxm@126.com

1 Institute for Infectious Disease and Endemic Disease Control, Beijing Center for Disease Prevention and Control, Beijing Center for Prevention Medical Research, Beijing Key Laboratory of Diagnostic and Traceability Technologies for Food Poisoning, Beijing 100013, China

Full list of author information is available at the end of the article
}

infections, causing pneumoniae, abscess, bacteremia, and urinary tract infections [1], and occasionally causes diarrhea in humans $[2,3]$.

The surveillance on diarrhea-syndrome outpatients included any age people in Beijing, involving 245 sentinel hospitals from all 16 districts. Stool specimens were collected from diarrhea-syndrome outpatients in sentinel hospitals [4], and tested the common diarrhea induced pathogens including rotavirus, norovirus, diarrheagenic Escherichia coli, Salmonella, and Shigella spp. [5]. Because the 
increasingly nosocomial infections caused by $K$. pneumoniae might impose an increasing risk of infections in communities, from 2010 to 2015, K. pneumoniae testing was included into the exiting diarrhea-syndrome surveillance with objective to estimate the prevalence of $K$. pneumoniae in diarrheasyndrome outpatients, test antibiotics susceptibility and investigate microbiological characteristics.

\section{Methods}

\section{Identification of bacterial strains}

Stool specimens from diarrhea-syndrome outpatients were collected through Beijing diarrhea-syndrome surveillance from 2010 to 2015. Diarrheagenic bacteria strains were cultured and isolated from stool specimens firstly. Any isolated positive bacteria strains were further identified the pathogens (e.g., Salmonella, Shigella spp., diarrheagenic Escherichia coli, Vibrio parahemolyticus, and $K$. pneumoniae) by the Vitek2 Compact instrument (bioMérieux. Marcy, France). The isolated K. pneumoniae strains were proceeding for antibiotics susceptibility test and molecular characterization.

\section{Antibiotics susceptibility testing}

Antibiotics susceptibility testing for the $K$. pneumoniae strains was measured by minimal inhibitory concentration (MIC) method. The MICs were interpreted by the standards of Clinical and Laboratory Standards Institute (CLSI) document M100-S26:2016 [6]. The following 14 antibiotics sourced from Shanghai Xingbai Co. (AST panel for aerobic gram negative bacilli) were used for antimicrobial susceptibility test: ciprofloxacin, ampicillin, amoxicillinclavulanate, chloramphenicol, sulfisoxazole, trimethoprimsulfamethoxazole, nalidixic acid, ceftriaxone, gentamicin, tetracycline, cefotaxime, cefoxitin, cefepime, imipenem. Escherichia coli strain ATCC 25922 was in use as qualitycontrol strains for the antibiotics susceptibility testing. MIC level at or above $2 \mu \mathrm{g} / \mathrm{mL}$ for cefotaxime and ceftriaxone indicates the strains possibly produced extended-spectrum beta-lactamases (ESBL), which needs further confirmation. MIC for ceftazidime combination with clavulanate decrease at least three twofold concentration in contrast with the MIC value for ceftazidime alone (e.g., ceftazidime $\mathrm{MIC}=8 \mu \mathrm{g} / \mathrm{mL}$; ceftazidime-clavulanate MIC $=1 \mu \mathrm{g} / \mathrm{mL}$ ) confirms the existing ESBL production strains [6].

\section{Pulse field gel electrophoresis}

K. pneumoniae strains were subtyped by pulse field gel electrophoresis (PFGE) method using restriction endonucleases $X b a \mathrm{I}$ [7]. The restriction endonuclease $X b a \mathrm{I}$ (Fermentas) was used to digest the prepared genomic DNA and resultant DNA fragments were separated in a PFGE CHEF-DR III system (Bio-Rad Laboratories) in 0.5 $\times$ Tris-borate-EDTA buffer at $120 \mathrm{~V}$ for $18.5 \mathrm{~h}$, with pulse times ranging from 6 to
$36 \mathrm{~s}$. PFGE dendrogram was generated by BioNumerics version 7.1 (Applied Math, Belgium) with the unweighted pairgroup method (UPGMA) and Dice coefficient. Isolates that exhibited a PFGE profile with more than $80 \%$ similarity were considered as closely related strains $[8,9]$.

\section{Multilocus sequence typing}

Multilocus sequence typing (MLST) was performed to subtype $K$. pneumoniae strains using seven housekeeping genes (gapA, infB, $m d h$, pgi, phoE, rpoB, and tonB) [10]. Seven gene fragments of the K. pneumoniae strains were amplified by PCR and sequenced. The allele sequences and sequence types (STs) were determined by the Institute Pasteur Klebsiella MLST database (http://bigsdb.web. pasteur.fr/klebsiella/klebsiella.html). BioNumerics version 7.1 software was used to create the minimum spanning tree. In the minimum spanning tree, the founder ST was defined as the greatest number of single-locus variants. Types were represented by circles and the size of a circle indicated the number of strains with this particular type.

\section{Results}

Identification of bacterial strains

In the 4340 stool specimens collected in the surveillance period from 2010 to 2015, $22 \mathrm{~K}$. pneumoniae strains were identified: one strain in 2010, three strains in 2011 and 2012 respectively, five strains in 2013, nine strains in 2014, and one strain in 2015; 16 out of 22 positive K. pneumoniae were isolated from April to October, while six positives identified in low-incidence seasons 2010-2015.

\section{Antibiotics susceptibility results}

Antibiotics susceptibility is shown in Table 1 . The result showed that $22 \mathrm{~K}$. pneumoniae strains were all sensitive

Table 1 Antibiotics susceptibility results of 22 K. pneumoniae strains

\begin{tabular}{llll}
\hline Antibiotics agent & R (\%) & I (\%) & S (\%) \\
\hline Ampicillin & $16(72.7 \%)$ & $6(27.3 \%)$ & 0 \\
Amoxicillin-clavulanate & $22(100 \%)$ & 0 & 0 \\
Ceftriaxone & 0 & 0 & $22(100 \%)$ \\
Cefotaxime & 0 & 0 & $22(100 \%)$ \\
Cefepime & 0 & 0 & $22(100 \%)$ \\
Cefoxitin & $1(4.5 \%)$ & 0 & $21(95.5 \%)$ \\
Imipenem & 0 & 0 & $22(100 \%)$ \\
Tetracycline & $2(9.1 \%)$ & $7(31.8 \%)$ & $13(59.1 \%)$ \\
Nalidixic acid & 0 & 0 & $22(100 \%)$ \\
Ciprofloxacin & 0 & 0 & $22(100 \%)$ \\
Trimethoprim-sulfamethoxazole & $1(4.5 \%)$ & 0 & $21(95.5 \%)$ \\
Sulfisoxazole & $8(39.3 \%)$ & 0 & $14(63.6 \%)$ \\
Chloramphenicol & $4(18.2 \%)$ & $7(31.8 \%)$ & $11(50 \%)$ \\
Gentamicin & 0 & 0 & $22(100 \%)$ \\
\hline
\end{tabular}

$R$ resistant, / intermediate, $S$ susceptible 
to gentamicin, nalidixic acid, ciprofloxacin, ceftriaxone, cefotaxime, cefepime, imipenem, followed by $95.5 \%$ to trimethoprim-sulfamethoxazole, cefoxitin, and $63.6 \%$ to sulfisoxazole. The highest resistance rate of K. pneumoniae strains was $100 \%$ to amoxicillin-clavulanate, and followed by $72.7 \%$ to ampicillin. Moreover, all $22 \mathrm{~K}$. pneumoniae strains were sensitive to ceftriaxone, cefotaxime, the MIC was less than $1 \mu \mathrm{g} / \mathrm{mL}$, and no ESBL production strain was identified.

\section{PFGE typing}

22 isolates showed 21 different PFGE types. The dendrogram of the PFGE image showed that four K. pneumoniae strains isolated during 2011-2014 had over $80 \%$ similarity, which categorized into one PFGE cluster (cluster A), while other strains had less similarity (Fig. 1).

\section{MLST typing}

MLST was performed for all 22 isolates to investigate the genetic relationships. MLST analysis revealed 20 different sequence types (STs), including three ST23 strains and one ST65. In addition to identifying ST23 and ST65 which are prevail in Asia countries, ST2362, ST2363, ST2364, ST2365, ST2366, ST2367, ST2368, ST2369, and ST2370 were discerned for the first time. In the minimum spanning tree, there were two clone groups: one clone group contained ST23 and ST1660, and the other clone group contained ST17 and ST20 (Fig. 2).

\section{Discussion}

K. pneumoniae is one of the most common Gram-negative pathogen found in human's nasopharynx and in the intestinal tract [1]. It existed with $60-70 \%$ the carrier rate

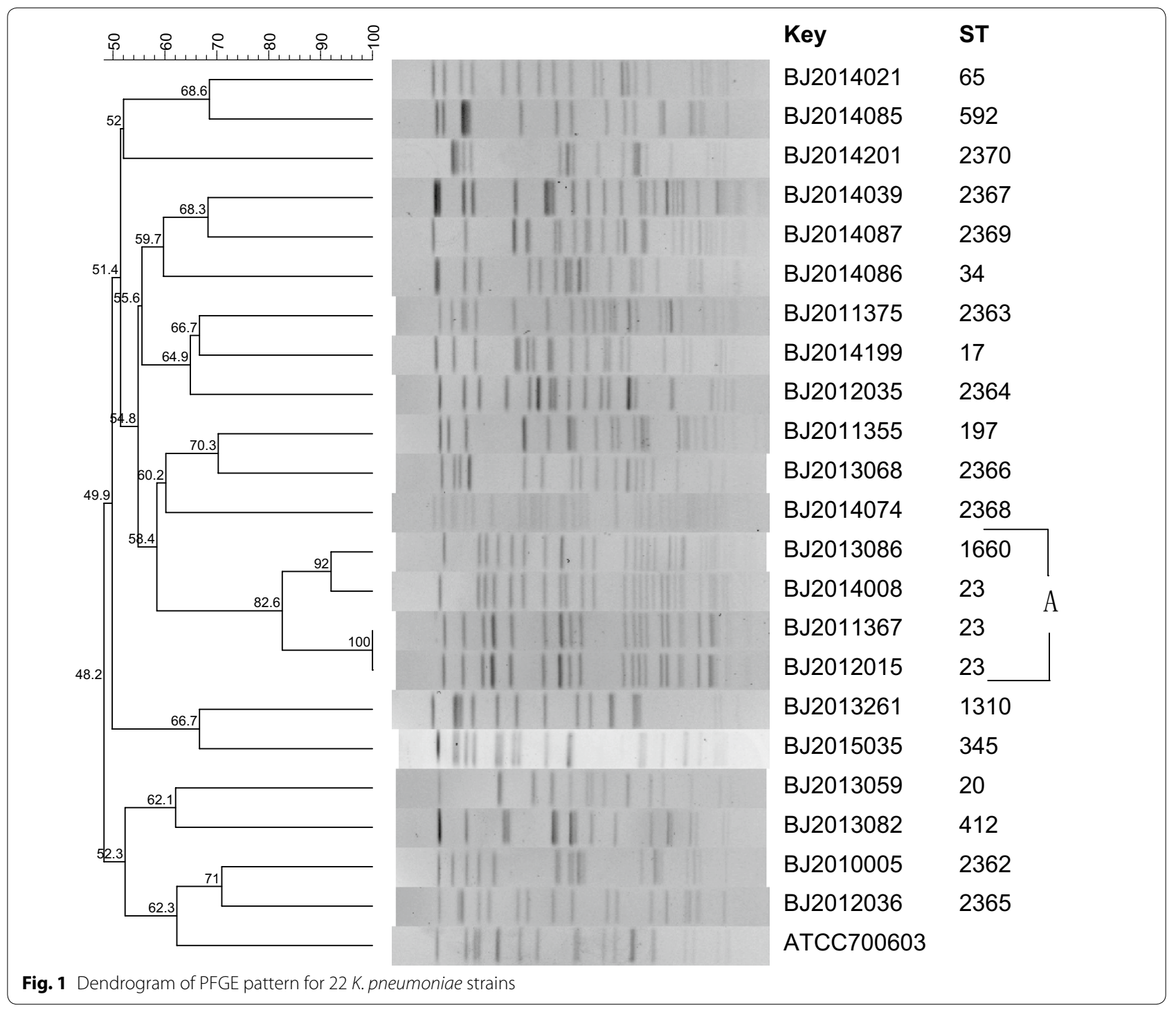


in the hospital environment, and was notified as a common pathogen caused nosocomial infection [1]. However, the community-acquired $K$. pneumoniae infection was rare reported. This study, through inclusion of K. pneumoniae into the existing diarrhea-syndrome surveillance, was able to detect $K$. pneumoniae infection in community and its risk for possible persistent transmission in population in Beijing.

The detection rate of $K$. pneumoniae in stool specimens from outpatients with diarrhea syndromes was about $0.5 \%(22 / 4340)$, which further demonstrated the existence of the community-acquired $K$. pneumoniae infection [11]. The prevalence in this study was much lower than the previous detection rate of $K$. pneumoniae in stool samples ranges from 5 to $38 \%$ in hospital patients [11], which emphasized the importance of nosocomial infection control as well necessary vigilance for detecting community-acquired $K$. pneumoniae.

Less similarity of the strains typed by PFGE or MLST indicated the unlikely long-term transmission

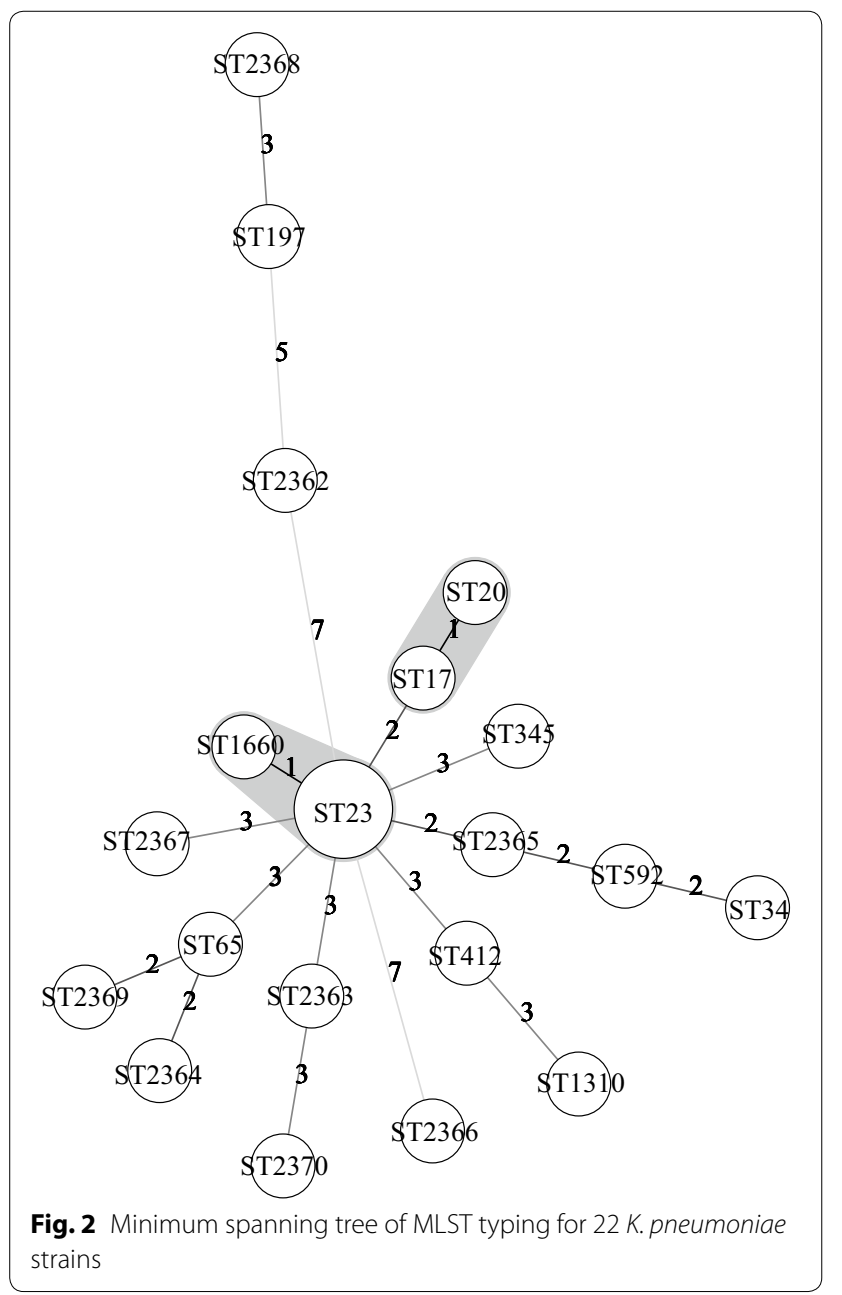

of K. pneumoniae in the community. This study identified ST23 as the most common genotype, which were reported in many previous hospital-acquired infection studies in Asia $[12,13]$. Noticeable this study isolated one ST65 strain, a mucoid phenotype and harbored rmpA gene for aerobactin, which more likely caused community-acquired infection [14, 15] and indicated as one of the independent risk factors for bacteremia in patients with pneumonia [13]. Moreover, the microbiological character of newly detected ST2362, ST2363, ST2364, ST2365, ST2366, ST2367, ST2368, ST2369, and ST2370, these strains presented clinical character are not thoroughly explicit, which needs further studies.

No ESBL production strains existed from stool specimens of diarrhea-syndrome outpatients in recent years illustrated that the community-acquired $K$. pneumoniae was not a serious public health problem. All the strains were sensitive to some antibiotics (e.g., cephalosporins, quinolones, and fluoroquinolones) universally in use for clinical treatment. However, hospital-acquired K. pneumoniae infection with resistance to multiple antibiotics agents has been increasing [16]. The Klebsiella species strains (e.g., TEM-type and SHV-type ESBLs, CTX-M type ESBLs) caused several predominant nosocomial infections [17]. This study did not identify the drug resistance for $K$. pneumoniae strains, but the diarrhea-syndrome surveillance identified serious drug resistance for Shigella spp. [4]. Given the consideration of horizontal transfer of drug resistance related genes as one of the most important mechanisms for the dramatically quickly dissemination of multi drug resistance among bacteria [18], monitoring the drug resistance of the community-acquired $K$. pneumoniae strains can provide a significant signal of drug resistance in population.

\section{Conclusions}

The detection rate of K. pneumoniae in stool specimens from outpatients with diarrhea syndromes was about $0.5 \%$ in Beijing. Less similarity of the isolated strains indicated the unlikely long-term transmission of K. pneumoniae in the community. ST23 was the most common genotype. Drug resistance of the community-acquired $K$. pneumoniae was not a serious problem in comparing with hospital-acquired infections. High vigilance in the drug resistance of the community-acquired $K$. pneumoniae strains can provide a significant signal of drug resistance in population.

\section{Abbreviations}

CLSI: Clinical and Laboratory Standards Institute; ESBL: extended-spectrum beta-lactamases; K. pneumoniae: Klebsiella pneumoniae; MIC: minimum inhibitory concentration; MLST: multilocus sequence typing; PFGE: pulsed field gel electrophoresis; STs: sequence types; UPGMA: the unweighted pair-group method. 


\section{Authors' contributions}

BL participated in molecular genetic studies and drafted the manuscript; XZ and $\mathrm{YH}$ participated in sample collection and antimicrobial susceptibility testing; $\mathrm{HZ}$ carried out the data analysis; $\mathrm{MQ}$ and $\mathrm{QW}$ participated in the design of the study. All authors read and approved the final manuscript.

\section{Author details}

${ }^{1}$ Institute for Infectious Disease and Endemic Disease Control, Beijing Center for Disease Prevention and Control, Beijing Center for Prevention Medical Research, Beijing Key Laboratory of Diagnostic and Traceability Technologies for Food Poisoning, Beijing 100013, China. ${ }^{2}$ State Key Laboratory for Infection Disease Prevention and Control, National Institute for Communicable Disease Prevention and Control, Chinese Center for Disease Control and Prevention, Beijing 102206, China. ${ }^{3}$ Collaborative Innovation Center for Diagnosis and Treatment of Infectious Diseases, Hangzhou, China.

\section{Acknowledgements}

We thank the team of the curators of the Institute Pasteur MLST system (Paris, France) for importing novel alleles, profiles and/or isolates at http://bigsdb. web.pasteur.fr.

\section{Competing interests}

The authors declare that they have no competing interests.

\section{Availability of data and materials}

The datasets used and/or analysed during the current study are available from the corresponding author on reasonable request.

\section{Consent for publication}

This manuscript did not include any details, images, or videos relating to individual participants.

\section{Ethics approval and consent to participate}

The study was approved by the Ethics Committee of the Beijing Center for Disease Control and Prevention. All patients in the study signed informed consent forms.

\section{Funding}

This work was supported by the Project of Beijing High-Level Technical Personnel Training in Health (2013-3-099) and National Science and Technology Support Projects for the "Thirteenth Five-Year Plan" for Infectious Diseases of China (2017ZX10104002-003-002).

\section{Publisher's Note}

Springer Nature remains neutral with regard to jurisdictional claims in published maps and institutional affiliations.

Received: 28 March 2017 Accepted: 20 June 2017

Published online: 30 June 2017

\section{References}

1. Podschun R, Ullmann U. Klebsiella spp. as nosocomial pathogens: epidemiology, taxonomy, typing methods, and pathogenicity factors. Clin Microbiol Rev. 1998;11:589-603 (Epub 1998/10/10).

2. Guerin F, Le Bouguenec C, Gilquin J, Haddad F, Goldstein FW. Bloody diarrhea caused by Klebsiella pneumoniae: a new mechanism of bacterial virulence? Clin Infect Dis. 1998:27:648-9 (Epub 1998/10/14)

3. Gassama-Sow A, Diallo MH, Wane AA, Seck A, Samb-Ba B, Sow PS, AidaraKane A. Genetic determinants of antibiotic resistance in diarrheagenic
Klebsiella pneumoniae subspecies ozaenae: an emerging enteropathogen in Senegal. Clin Infect Dis. 2010;50:453-5 (Epub 2010/01/13).

4. Qu M, Zhang X, Liu G, Huang Y, Jia L, Liang W, Li X, Wu X, Li J, Yan H, Kan B, Wang Q. An eight-year study of Shigella species in Beijing, China: serodiversity, virulence genes, and antimicrobial resistance. J Infect Dev Ctries. 2014;8:904-8 (Epub 2014/07/16).

5. Yu J, Jing H, Lai S, Xu W, Li M, Wu J, Liu W, Yuan Z, Chen Y, Zhao S, Wang X, Zhao Z, Ran L, Wu S, Klena JD, Feng L, Li F, Ye X, Qiu Y, Yu H, Li Z, Yang W. Etiology of diarrhea among children under the age five in China: results from a five-year surveillance. J Infect. 2015;71:19-27 (Epub 2015/03/11).

6. Performance standards for antimicrobial susceptibility testing. 26th ed. Wayne, PA 19087 USA: Clinical and Laboratory Standards Institute.

7. Ribot EM, Fair MA, Gautom R, Cameron DN, Hunter SB, Swaminathan B, Barrett TJ. Standardization of pulsed-field gel electrophoresis protocols for the subtyping of Escherichia coli O157:H7, Salmonella, and Shigella for PulseNet. Foodborne Pathog Dis. 2006;3:59-67 (Epub 2006/04/11).

8. Tseng IL, Liu YM, Wang SJ, Yeh HY, Hsieh CL, Lu HL, Tseng YC, Mu JJ. Emergence of carbapenemase producing Klebsiella pneumonia and Spread of KPC-2 and KPC-17 in Taiwan: a Nationwide Study from 2011 to 2013. PLoS ONE. 2015;10:e0138471 (Epub 2015/09/19).

9. Cheng L, Cao XL, Zhang ZF, Ning MZ, Xu XJ, Zhou W, Chen JH, Zhang $\mathrm{JH}$, Shen $\mathrm{H}$, Zhang K. Clonal dissemination of KPC-2 producing Klebsiella pneumoniae ST11 clone with high prevalence of oqXAB and rmtB in a tertiary hospital in China: results from a 3-year period. Ann Clin Microbiol Antimicrob. 2016;15:1 (Epub 2016/01/21).

10. Diancourt L, Passet V, Verhoef J, Grimont PA, Brisse S. Multilocus sequence typing of Klebsiella pneumoniae nosocomial isolates. J Clin Microbiol. 2005;43:4178-82 (Epub 2005/08/06).

11. Lee JC, Lee NY, Lee HC, Huang WH, Tsui KC, Chang CM, Lee CC, Chen PL, Wu CJ, Hsueh PR, Ko WC. Clinical characteristics of urosepsis caused by extended-spectrum beta-lactamase-producing Escherichia coli or Klebsiella pneumonia and their emergence in the community. J Microbiol Immunol Infect. 2012;45:127-33 (Epub 2011/11/02).

12. Qi Y, Wei Z, Ji S, Du X, Shen P, Yu Y. ST11, the dominant clone of KPCproducing Klebsiella pneumoniae in China. J Antimicrob Chemother. 2011;66:307-12 (Epub 2010/12/07).

13. Ito R, Shindo Y, Kobayashi D, Ando M, Jin W, Wachino J, Yamada K, Kimura K, Yagi T, Hasegawa Y, Arakawa Y. Molecular epidemiological characteristics of Klebsiella pneumoniae associated with bacteremia among patients with pneumonia. J Clin Microbiol. 2015;53:879-86 (Epub 2015/01/09).

14. Yu WL, Ko WC, Cheng KC, Lee HC, Ke DS, Lee CC, Fung CP, Chuang YC. Association between rmpA and magA genes and clinical syndromes caused by Klebsiella pneumoniae in Taiwan. Clin Infect Dis. 2006;42:1351-8 (Epub 2006/04/19)

15. Yu VL, Hansen DS, Ko WC, Sagnimeni A, Klugman KP, von Gottberg A, Goossens H, Wagener MM, Benedi VJ. Virulence characteristics of Klebsiella and clinical manifestations of K. pneumoniae bloodstream infections. Emerg Infect Dis. 2007;13:986-93 (Epub 2008/01/25).

16. Paterson DL, Ko WC, Von Gottberg A, Mohapatra S, Casellas JM, Goossens H, Mulazimoglu L, Trenholme G, Klugman KP, Bonomo RA, Rice LB, Wagener MM, McCormack JG, Yu VL. International prospective study of Klebsiella pneumoniae bacteremia: implications of extended-spectrum beta-lactamase production in nosocomial infections. Ann Intern Med. 2004;140:26-32 (Epub 2004/01/07).

17. Livermore DM. Current epidemiology and growing resistance of Gram-negative pathogens. Kor J Intern Med. 2012;27:128-42 (Epub 2012/06/19).

18. Canton R, Coque TM, Baquero F. Multi-resistant gram-negative bacilli: from epidemics to endemics. Curr Opin Infect Dis. 2003;16:315-25 (Epub 2003/07/16). 